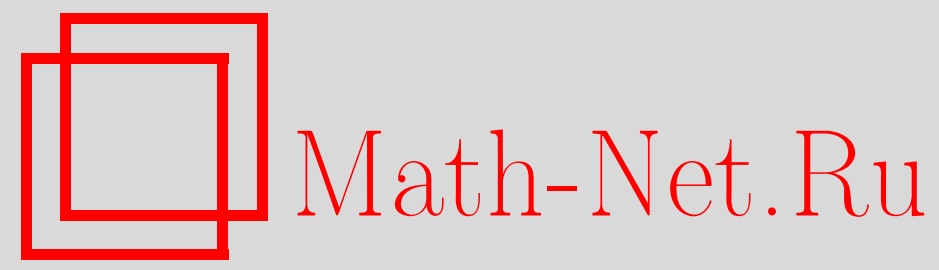

А. Н. Старцев, О распределении размера эпидемии в одной немарковской модели, Теория вероятн. и ее примен., 1996, том 41, выпуск 4, 827-839

DOI: https://doi.org/10.4213/tvp3204

Использование Общероссийского математического портала Math-Net.Ru подразумевает, что вы прочитали и согласны с пользовательским соглашением http://www . mathnet.ru/rus/agreement

Параметры загрузки:

IP: 3.85 .7 .115

26 апреля 2023 г., 18:14:04

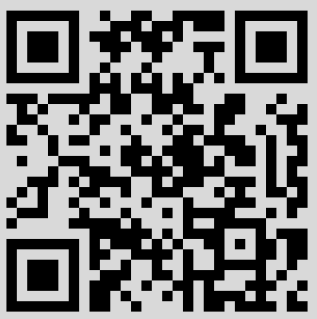




\section{О РАСПРЕДЕЛЕНИИ РАЗМЕРА ЭПИДЕМИИ В ОДНОЙ НЕМАРКОВСКОЙ МОДЕЛИ}

Получены точные и асимптотические распределения для размера эпидемии, развивающейся в замкнутой однородно смешивающейся популяции, когда заразные периоды носителей инфекции и уровни сопротивляемости (иммунитета) восприимчивых задаются независимыми случайными величинами при различных предположениях относительно их распределений.

Ключевые слова и фразы: распределение размера эпидемии, немарковские модели, порядковые статистики, предельные теоремы.

1. Введение. Две наиболее популярные марковские модели эпидемии задаются вероятностями изменения состояния популяции за бесконечно малый промежуток времени $(t, t+d t)$, отражаюшими процессы заболевания восприимчивых и устранения источников инфекции. Если $s$ - число восприимчивых, а $i$ - число носителей инфекции в момент $t$, то эти вероятности имеют вид

$$
\left\{\begin{array}{l}
\mathbf{P}\{(s, i) \longrightarrow(s-1, i+a)\}=s i d t+o(d t) \\
\mathbf{P}\{(s, i) \longrightarrow(s, i-1)\}=\rho i d t+o(d t)
\end{array}\right.
$$

При $\boldsymbol{a}=1$ получим так называемую общую вероятностную модель, или процесс Бартлетта-Мак-Кендрика (см. [1]). Если же $a=0$, то (1) задает модель Вейса [2], в которой вновь зараженные восприимчивые не являются источниками инфекции; они опознаются (ввиду явных симптомов) и устраняются из популяции.

Отметим также, что модели типа (1) являются частными случаями процессов с взаимодействием частиц, введенных Б. А. Севастьяновым в 1981 г. [3]. Для этих пропессов были получены соответствующие уравнения Колмогорова для производящей функции вероятностей состояний, которые, однако, в обшем случае не разрепимы в явном виде.

Для частного случая модели (1) с $a=1$ для соответствующего уравнения

$$
\frac{\partial \Pi_{t}(x, y)}{\partial t}=x(x-y) \frac{\partial^{2} \Pi_{t}(x, y)}{\partial x \partial y}+\rho(1-x) \frac{\partial \Pi_{t}(x, y)}{\partial x}, \quad \Pi_{0}(x, y)=x^{m} y^{n}
$$

*Институт математики АН Узбекистана, ул. Ф. Ходжаева, 29, 700143 Ташкент, Узбекистан. 
Гани [4] и, независимо от него, Сискинд [5] в 1965 г. нашли решение в явной форме и тем самым получили точное, но необозримо громоздкое аналитическое выражение для вероятностей $p_{t}(s, i)$ состояний процесса в любой заданный момент времени $t$.

Это обстоятельство стимулировало получение асимптотических результатов для некоторых наиболее характерных функционалов от рассматриваемого процесса. К числу таковых относятся такие функционалы, как размер эпидемии, ее продолжительность и стоимость. Асимптотический подход базируется на предположении, что первоначальное число восприимчивых $n \rightarrow \infty$, а исходное число носителей инфекции $m$ и параметр $\rho$ могут меняться вместе с $n$ («схема серий»).

При этом большее внимание уделялось исследованию размера эпидемии $\nu$, т.е. числу переболевших восприимчивых к моменту окончания эпидемии. С формальной точки зрения, $\nu$ соответствует поглошению процесса с переходными вероятностями (1) в состоянии $(n-\nu, 0)$.

Заметим, что для получения распределения размера эпидемии $\nu$ достаточно ограничиться рассмотрением вложенной цепи Маркова, соответствующей процессу (1), переходные вероятности которой имеют вид

$$
\left\{\begin{array}{l}
\mathbf{P}\{(s, i) \longrightarrow(s-1, i+a)\}=p_{s} \equiv \frac{s}{s+\rho}, \\
\mathbf{P}\{(s, i) \longrightarrow(s, i-1)\}=q_{s} \equiv 1-p_{s} .
\end{array}\right.
$$

Вероятность $P_{k}=\mathbf{P}\{\nu=k\}$ как функция от свободных переменных $p_{s}$ может быть представлена в виде

$$
P_{k}=\left(\prod_{i=0}^{k-1} p_{n-i}\right) \sum_{D_{k, a}}\left(\prod_{j=0}^{k} q_{n-j}^{\alpha_{j}}\right),
$$

где $D_{k, a}=\left\{\left(i_{0}, \ldots, i_{k}\right): i_{0}+\cdots+i_{j}=m+a j, j=0, \ldots, k-1 ; i_{0}+\cdots+i_{k}=\right.$ $m+a k\}$.

В случае, когда $p_{s}=s /(s+\rho), s \geqslant 0$, и $a=0$, эта вероятность приводится к простому виду [2]:

$$
P_{k}=\left(\begin{array}{l}
n \\
k
\end{array}\right) \sum_{i=0}^{k}(-1)^{k-i}\left(\begin{array}{l}
k \\
i
\end{array}\right)\left[\frac{\rho}{n-i+\rho}\right]^{m} .
$$

Вудс [6] получил более простое по сравнению с (3) представление для вероятности $P_{k}$ при произвольных $p_{s}$ и $q_{s}$ в случае $a=0$.

Исходя из (3), получаем следуюшую систему линейных уравнений для вероятностей $P_{k}$ при произвольных $q_{n-s} \neq q_{n-k}, s=1, \ldots, k-1,[7]$ :

$$
\sum_{i=0}^{k} a_{k i} P_{i}=b_{k}, \quad k=0,1, \ldots, n,
$$


где

$$
\begin{aligned}
& a_{k i}=\left\{\begin{array}{ll}
1, & i=k, \\
\Pi_{k i} q_{n-k}^{a(k-i)}, & i<k,
\end{array} \quad \Pi_{k i}=\prod_{s=i}^{k-1} \frac{p_{n-s}}{1-q_{n-s} / q_{n-k}},\right. \\
& \Pi_{00}=1, \quad b_{k}=\Pi_{k 0} q_{n-k}^{m+a k} .
\end{aligned}
$$

Система (5) при $a=1$ и $p_{s}$ вида (2) была получена (довольно громоздким методом) еще в 1955 г. в работах Бейли [8] и Уитла [9]. В этом случае $\Pi_{k i}=\left(\begin{array}{c}n-i \\ k-i\end{array}\right)$.

Что касается продолжительности эпидемии $\tau$, то исследование этого функционала сводится к изучению сумм случайного числа показательно распределенных слагаемых, представляющих собой времена пребывания в состояниях исходного марковского процесса вплоть до поглощения, т.е.

$$
\tau=\sum \tau_{s i}
$$

где $\mathbf{P}\left\{\tau_{s i}>t\right\}=\exp (-i(s+\rho) t)$, а суммирование проводится по всем траекториям из начальното состояния $(n, m)$ до состояния поглощения $(n-\nu, 0)$. Из сказанного ясно, что этот функционал имеет достаточно сложную структуру.

Стоимость эпидемии $\eta$ представляет собой суммарные потери времени, вызванные эпидемией, и, с формальной точки зрения, представляет собой площадь под траекторией числа больных, т.е.

$$
\eta=\int_{0}^{\tau} I(t) d t
$$

где $I(t)$ - число больных в момент времени $t$.

Достаточно полный асимптотический анализ размера эпидемии в модели (1) при $a=1$ был проведен в работе А. В. Нагаева и автора [10]. Аналогичные исследования проведены также и в случае, когда первая из вероятностей перехода в (1) имеет вид $\varphi(s) i d t+o(d t)[11]$, что позволяет в какой-то степени учитывать неоднородность смешивания в популяциях.

Один из не исследованных в [10] случаев, когда эпидемия охватывает практически всю популяцию, за исключением конечного числа восприимчивых, технически наиболее сложный, был рассмотрен несколько позже в работе автора [12], где показано, что если $n \exp (-(m+n) / \rho) \longrightarrow \lambda$, To

$$
\lim _{n \rightarrow \infty} \mathbf{P}\{n-\nu=k\}=\frac{\lambda^{k}}{k !} e^{-\lambda}, \quad k \geqslant 0 .
$$

При этом были описаны и соответствуюшие переходные явления.

2. Конструкция Селке. Селке [13], исходя из результатов работы [10], получил соотношение (6) значительно позже, чем в [12], при этом он использовал другое представление модели (1). 
Конструкция Селке состоит в следующем. Вводятся независимые случайные величины (н.с.в.) $T_{1}, T_{2}, \ldots$, имеющие показательное распределение с параметром $\rho$ и интерпретируемые как инфекционные периоды источников инфекции. Затем рассматриваются независимые между собой и от $\left\{T_{i}, i \geqslant 1\right\}$ случайные величины $L_{1}, \ldots, L_{n}$, также с показательным распределением, но с параметром 1, интерпретируемые как уровень иммунитета (сопротивляемости) восприимчивых. Развитие эпидемии происходит следующим образом. Источники инфекции по истечении инфекционного периода устраняются из популяции (т.е. не являются ни источниками распространения, ни источниками заражения). Восприимчивые накапливают инфекцию от каждого из находяшихся в популяции заразных индивидуумов с единичной интенсивностью и, когда ее суммарное количество достигает их уровня сопротивляемости, они заражаются.

Эквивалентность модели (1) и введенной конструкции легко следует из свойства «отсутствия последействия» показательного распределения.

В конструкции Селке распределение размера эпидемии можно записать в следующем виде:

$$
P_{k} \equiv \mathbf{P}\{\nu=k\}=\mathbf{P}\left\{L_{(i)} \leqslant S_{m+a(i-1)}, i=1, \ldots, k ; L_{(k+1)}>S_{m+a k}\right\},
$$

где $S_{i}=T_{1}+\cdots+T_{i}$, а $L_{(1)} \leqslant L_{(2)} \leqslant \cdots \leqslant L_{(n)}$ - вариационный ряд.

Легко заметить, что в модели Вейса $(a=0)$ эта вероятность принимает более простой вид

$$
P_{k}=\mathbf{P}\left\{L_{(k)} \leqslant S_{m}, L_{(k+1)}>S_{m}\right\}
$$

В дальнейшем конструкция Селке стала использоваться и в случае непоказательных распределений $T_{i}$. В частности, в работе Болла [14] в случае $a=1$ было установлено тождество Вальда для эпидемических процессов: если $\left\{T_{i}\right\}$ и $\left\{L_{i}\right\}$ - независимые одинаково распределенные случайные величины, причем $L_{1}$ распределена показательно с параметром $1\left(L_{1} \sim \exp (1)\right)$, то

$$
\mathbf{E} \exp \left\{-t \eta(h(t))^{-m-\nu}\right\}=1,
$$

где $h(t)=\mathbf{E} \exp \left(-t T_{1}\right)$, а $\eta=T_{1}+T_{2}+\cdots+T_{m+\nu}-$ стоимость эпидемии.

Из этого тождества в [14] выведена система уравнений для вероятностей $P_{k}$ :

$$
\sum_{k=0}^{i}\left(\begin{array}{c}
n-k \\
i-k
\end{array}\right)[h(n-i)]^{-m-k} P_{k}=\left(\begin{array}{c}
n \\
i
\end{array}\right), \quad i=0,1, \ldots, n
$$

которая в случае, когда $T_{1}$ имеет показательное распределение с параметром $\rho\left(T_{1} \sim \exp (\rho)\right)$, сводится к соответствующей системе БейлиУитла (см. также (5)). 
3. Немарковский аналог модели Вейса. Точные распределения. Оказывается, что для конструкции Селке, являющейся аналогом модели (1) с $a=0$, можно получить явный вид вероятностей $P_{k}$ типа (4) при произвольных распределениях $T_{1}, T_{2}, \ldots, T_{m}$.

Положим $G_{i}(x)=\mathbf{P}\left\{T_{i}<x\right\}, h_{i}(t)=\mathbf{E} \exp \left(-t T_{i}\right)$.

Теорема 1. Если $T_{1}, \ldots, T_{m}$ - независимые случайные величины, а $L_{1}, \ldots, L_{n}-$ не зависящие от них независимые одинаково распределенные случайные величины с $L_{1} \sim \exp (1)$, по для любых $k=0,1, \ldots$

$$
P_{k}=\left(\begin{array}{l}
n \\
k
\end{array}\right) \sum_{i=0}^{k}(-1)^{k-i}\left(\begin{array}{c}
k \\
i
\end{array}\right) \prod_{j=1}^{m} h_{j}(n-i) .
$$

Eсли $T_{i} \sim \exp (\rho)$, mо $h_{i}(t)=\rho /(\rho+t)$, и из (8) следует результат Beŭca (4).

Д ок аз а те ль с тв о те о рем ы 1. Исходя из (7), заметим, что совместная плотность распределения порядковых статистик $L_{(k)}$ и $L_{(k+1)}$ в случае, когда $L_{1} \sim \exp (1)$, имеет вид [15]:

$$
p_{k, k+1}(u, v)=\frac{\Gamma(n+1)}{\Gamma(k) \Gamma(n-k)}\left(1-e^{-u}\right)^{k-1} e^{-u-(n-k) v} .
$$

Следовательно,

$$
\begin{aligned}
\mathbf{P} & \left\{L_{(k)} \leqslant y, L_{(k+1)}>y\right\} \\
& =\frac{\Gamma(n+1)}{\Gamma(k) \Gamma(n-k)} \int_{0}^{y}\left(1-e^{-y}\right)^{k-1} e^{-u} d u \int_{-\infty}^{\infty} e^{-(n-k) v} d v \\
& =\left(\begin{array}{c}
n \\
k
\end{array}\right) e^{-(n-k) y}\left(1-e^{-y}\right)^{k}=\left(\begin{array}{c}
n \\
k
\end{array}\right) e^{-(n-k) y} \sum_{i=0}^{k}(-1)^{i}\left(\begin{array}{c}
k \\
i
\end{array}\right) e^{-i y} .
\end{aligned}
$$

В силу этого имеем

$$
\begin{aligned}
P_{k} & =\int_{0}^{\infty} \cdots \int_{0}^{\infty} \mathbf{P}\left\{L_{(k)} \leqslant \sum_{i=1}^{m} u_{i}, L_{(k+1)}>\sum_{i=0}^{m} u_{i}\right\} d G_{1}\left(u_{1}\right) \cdots d G_{m}\left(u_{m}\right) \\
& =\left(\begin{array}{l}
n \\
k
\end{array}\right) \sum_{i=0}^{k}(-1)^{i}\left(\begin{array}{c}
k \\
i
\end{array}\right) \int_{0}^{\infty} \cdots \int_{0}^{\infty} \prod_{j=1}^{m} \exp \left(-(n-k+i) u_{j}\right) d G_{j}\left(u_{j}\right) \\
& =\left(\begin{array}{l}
n \\
k
\end{array}\right) \sum_{i=0}^{k}(-1)^{i}\left(\begin{array}{c}
k \\
i
\end{array}\right) \prod_{j=1}^{m} h_{j}(n-k+i) \\
& =\left(\begin{array}{l}
n \\
k
\end{array}\right) \sum_{i=0}^{k}(-1)^{k-i}\left(\begin{array}{c}
k \\
i
\end{array}\right) \prod_{j=1}^{m} h_{j}(n-i),
\end{aligned}
$$

что и требовалось доказать. 
4.1. Предельные распределения для локальных вероятностей. Крайние случаи. Без предположения о показательности распределений с.в. $L_{1}, \ldots, L_{n}$ можно получать асимптотические результаты, основываясь на предельных теоремах для порядковых статистик при $n \rightarrow \infty$, полученных в работах Б. В. Гнеденко [16], Н. В. Смирнова [17], [18] и других авторов.

При этом более полный асимптотический анализ удается провести в интегральной постановке, исходя из равенства

$$
\mathbf{P}\{\nu>k\}=\mathbf{P}\left\{L_{(k+1)} \leqslant S_{m}\right\},
$$

в некоторых случаях можно получать асимптотику и для локальных вероятностей (см. теоремы 2 и 3 ниже).

Естественно предположить, что распределения с.в. $T_{1}, \ldots, T_{m}$ зависят от $m$, а само $m$ меняется вместе с $n$. Относительно с.в. $L_{1}, \ldots, L_{n}$ будем предполагать, что они одинаково распределены.

Введем следующие обозначения:

$$
\begin{aligned}
a_{i} & \equiv a_{i}(m)=\mathbf{E} T_{i}, & & \sigma_{i}^{2} \equiv \sigma_{i}^{2}(m)=\mathbf{D} T_{i}, \\
G_{i}(x) & =\mathbf{P}\left\{T_{i}<x\right\}, & & i=1, \ldots, m, \quad A_{m}=a_{1}+\cdots+a_{m}, \\
B_{m}^{2} & =\sigma_{1}^{2}+\cdots+\sigma_{m}^{2}, & & F(x)=\mathbf{P}\left\{L_{1}<x\right\} .
\end{aligned}
$$

Далее, пусть $\alpha(F)=\inf (x: F(x)>0), \beta(F)=\sup (x: F(x)<1)-$ границы носителя распределения $F$. Ввиду неотрицательности с.в. $L_{1}$ ясно, что $0 \leqslant \alpha(F)<\infty$.

Введем также следуюшее условие: при $m \rightarrow \infty$

$$
L_{m} \equiv B_{m}^{-2} \sum_{i=1}^{m} \int_{x-a_{i} \geqslant \varepsilon B_{m}}\left(x-a_{i}\right)^{2} d G_{i}(x) \longrightarrow 0 \quad \text { при любом } \varepsilon>0 .
$$

Теорема 2. Пусть выполнено условие (L) и существует такое $\gamma>0$, что для любого $x \in \mathbf{R}^{+}$

$$
\lim _{t \rightarrow 0} \frac{F(\alpha(F)+t / x)}{F(\alpha(F)+t)}=x^{-\gamma}
$$

пусть также выполнено одно из следующих условий: при $n \rightarrow \infty$

$1^{\circ} . \quad B_{m} d_{n}^{-1} \rightarrow b<\infty,\left[A_{m}-\alpha(F)\right] d_{n}^{-1} \longrightarrow c,|c|<\infty$,

$2^{\circ} . \quad B_{m} d_{n}^{-1} \rightarrow \infty,\left[A_{m}-\alpha(F)\right] B_{m}^{-1} \longrightarrow c,|c|<\infty$,

где $d_{n}=\sup (x: F(x) \leqslant 1 / n)-\alpha(F)$.

Тогда для любого фиксированного $k \geqslant 0$

$$
\mathbf{P}\{\nu=k\} \rightarrow\left\{\begin{array}{l}
\delta_{0 k} \Phi\left(-\frac{c}{b}\right)+\int_{0}^{\infty} \Pi_{k}\left(x^{\gamma}\right) d \Phi\left(\frac{x-c}{b}\right) \\
\delta_{0 k}[1-\Phi(c)], \quad \text { если выполняется } 1^{\circ} \\
\text { если выполняется } 2^{\circ},
\end{array}\right.
$$


где $\Pi_{k}(\lambda)=\left(\lambda^{k} / k !\right) e^{-\lambda}, \Phi(x)=(2 \pi)^{-1 / 2} \int_{-\infty}^{x} e^{-u^{2} / 2} d u, \delta_{i j}-$ символ Кронекера.

Следствие 2.1. Если выполнено условие $1^{\circ}$ u $b=0$, то для любого $k \geqslant 0$

$$
\mathbf{P}\{\nu=k\} \longrightarrow \begin{cases}\Pi_{k}\left(c^{\gamma}\right), & c>0 \\ \delta_{0 k}, & c \leqslant 0\end{cases}
$$

Следствие 2.2. Если $T_{i} \sim \exp (\rho), L_{i} \sim \exp (1) u m n / \rho \rightarrow c$, mo $\mathbf{P}\{\nu=k\} \longrightarrow \Pi_{k}(c), k=0,1, \ldots$

Д о к а з а т ль с т о т е о ем ы 2. Будем исходить из соотношения (9) и воспользуемся предельными теоремами для порядковых статистик из монографии Я. Галамбоша [19]. В частности, из теоремы 2.8 .2 [19] следует, что при любом фиксированном $k>1$

$$
L_{k}^{*} \equiv\left(L_{(k)}-c_{n}\right) d_{n}^{-1} \stackrel{d}{\longrightarrow} \xi_{k} \Longleftrightarrow L_{(1)}^{*} \stackrel{d}{\longrightarrow} \xi
$$

где

$$
c_{n}=\alpha(F), \quad d_{n}=\sup \left(x: F(x) \leqslant \frac{1}{n}\right)-\alpha(F),
$$

причем для $x \in\left(\alpha\left(F_{\xi}\right), \beta\left(F_{\xi}\right)\right)$

$$
F_{\xi_{k}}(x) \equiv \mathbf{P}\left\{\xi_{k}<x\right\}=1-\left(1-F_{\xi}(x)\right) \sum_{i=0}^{k-1} \frac{\left[-\log \left(1-F_{\xi}(x)\right)\right]^{i}}{i !}
$$

Цалее, из теоремы 2.1.4 [19] следует, что в наших условиях

$$
F_{\xi}(x) \equiv \mathbf{P}\{\xi<x\}= \begin{cases}1-\exp \left(-x^{\gamma}\right), & x>0 \\ 0, & x \leqslant 0\end{cases}
$$

Соотношение (9) перепишем в виде

$$
\mathbf{P}\{\nu>k\}=\mathbf{P}\left\{L_{(k+1)}^{*} \leqslant S_{m}^{*} B_{m} d_{n}^{-1}+\left(A_{m}-c_{n}\right) d_{n}^{-1}\right\}
$$

где $S_{m}^{*}=\left(S_{m}-A_{m}\right) B_{m}^{-1}$, а $c_{n}$ и $d_{n}$ выбраны согласно (11).

Отсюда, из условия $1^{\circ}$, соотношения (10) и центральной предельной теоремы следует, что

$$
\mathbf{P}\{\nu>k\} \longrightarrow \mathbf{P}\left\{\xi_{k+1} \leqslant b \eta+c\right\}
$$

где $F_{\eta}(x)=\Phi(x)$.

Из (12) и (13) получим

$$
\mathbf{P}\left\{\xi_{k+1}<x\right\}= \begin{cases}1-\exp \left(-x^{\gamma}\right) \sum_{i=0}^{k} \frac{\left(x^{\gamma}\right)^{i}}{i !}, & x>0 \\ 0, & x \leqslant 0 .\end{cases}
$$


Теперь (15) можно переписать в виде

$$
\begin{aligned}
\mathbf{P}\{\nu \leqslant k\} & \longrightarrow \Phi\left(-\frac{c}{b}\right)+\int_{-c / b}^{\infty} \mathbf{P}\left\{\xi_{k+1} \geqslant b x+c\right\} d \Phi(x) \\
& =\Phi\left(-\frac{c}{b}\right)+\sum_{i=0}^{k} \int_{0}^{\infty} \Pi_{i}\left(x^{\gamma}\right) d \Phi\left(\frac{x-c}{b}\right) .
\end{aligned}
$$

Откуда и следует утверждение теоремы при выполнении условия $1^{\circ}$, если учесть, что $\mathbf{P}\{\nu=0\} \longrightarrow \Phi(-c / b)+\int_{0}^{\infty} \Pi_{0}\left(x^{\gamma}\right) d \Phi((x-c) / b)$.

Если же выполнено условие $2^{\circ}$, то, исходя из (14), можно записать соотношение

$$
\mathbf{P}\{\nu>k\}=\mathbf{P}\left\{L_{(k+1)}^{*} d_{n} B_{m}^{-1} \leqslant S_{m}^{*}+\left(A_{m}-c_{n}\right) B_{m}^{-1}\right\},
$$

из которого в результате предельного перехода получим: для любого $k \geqslant 1$

$$
\mathbf{P}\{\nu>k\} \longrightarrow \mathbf{P}\{\eta \geqslant-c\}=\Phi(c) .
$$

Д ок а за т ел ь с т о сл е д с т и я 2.2. Легко показать, что в условиях следствия

$$
c_{n}=\alpha(F)=0, \quad d_{n}=-\log \left(1-\frac{1}{n}\right) \sim \frac{1}{n}, \quad \gamma=1
$$

и $\left(A_{m}-c_{n}\right) d_{n}^{-1} \sim m n / \rho$. Если теперь $m n / \rho \rightarrow c$, то $B_{m} d_{n}^{-1}=n \sqrt{m} / \rho=$ $O\left(m^{-1 / 2}\right)=o(1)$, т.е. $b=0$. Далее, нетрудно проверить, что отношение Линдеберга оценивается следующим образом:

$$
L_{m}=O\left(m e^{-\varepsilon \sqrt{m}}\right)=o(1)
$$

и требуемый результат вытекает из следствия 2.1 .

Теорема 3. Пусть $\int_{\beta_{0}}^{\beta(F)}(1-F(y)) d y<\infty$ для некоторого конечного $\beta_{0}$ u

$$
\lim _{t \rightarrow \beta(F)} \frac{1-F(t+x R(t))}{1-F(t)}=e^{-x} \quad \text { для любого } \quad x \in \mathbf{R}
$$

где $R(t)=(1-F(t))^{-1} \int_{t}^{\beta(F)}(1-F(y)) d y$ npu $t \in(\alpha(F), \beta(F))$.

Пусть, кроме того, выполнено (L) и одно из следующих условий:

$1^{\circ} . \quad B_{m} d_{n}^{-1} \rightarrow b, 0 \leqslant b<\infty,\left(A_{m}-c_{n}\right) d_{n}^{-1} \longrightarrow c,|c|<\infty$,

$2^{\circ} . \quad B_{m} d_{n}^{-1} \rightarrow \infty,\left(A_{m}-c_{n}\right) B_{m}^{-1} \longrightarrow c,|c|<\infty$, при $n \rightarrow \infty$, где $c_{n}=\inf (x: 1-F(x) \leqslant 1 / n) u d_{n}=R\left(c_{n}\right)$. Tогда при $n \rightarrow \infty$ для любого $k \geqslant 0$

$$
\mathbf{P}\{\nu=k\} \longrightarrow \begin{cases}\int_{\infty}^{+\infty} \Pi_{k}\left(e^{-c-b x}\right) d \Phi(x), & \text { если выполнено } 1^{\circ}, \\ \delta_{n k} \Phi(c), & \text { если выполнено } 2^{\circ}\end{cases}
$$


где, как и ранее, $\Pi_{k}(\lambda)$-- распределение Пуассона с параметром $\lambda$.

Следствие 3.1. Eсли $T_{i} \sim \exp (\rho), L_{i} \sim \exp (1), \log n=o(\rho)$, $n \exp (-m / \rho) \longrightarrow \lambda, m o$

$$
\mathbf{P}\{n-\nu=k\} \longrightarrow \Pi_{k}(\lambda), \quad k=0,1, \ldots
$$

Доказ а те ль с т в о те о рем ы 3 . Из теоремы 2.8 .1 в [19] следует, что в условиях теоремы

$$
L_{(n-k+1)}^{*} \equiv\left(L_{(n-k+1)}-c_{n}\right) d_{n}^{-1} \stackrel{d}{\longrightarrow} \xi_{k} \Longleftrightarrow L_{(n)}^{*} \stackrel{d}{\longrightarrow} \xi,
$$

причем для $x \in\left(\alpha\left(F_{\xi}\right), \beta\left(F_{\xi}\right)\right)$

$$
F_{\xi_{k}}(x)=F_{\xi}(x) \sum_{i=0}^{k-1} \frac{\left[-\log F_{\xi}(x)\right]^{i}}{i !} .
$$

Далее, как следует из теоремы 2.1.3 [19],

$$
F_{\xi}(x)=\exp \left(e^{-x}\right), \quad x \in \mathbf{R},
$$

а нормирующие последовательности $c_{n}$ и $d_{n}$ можно определить равенствами

$$
c_{n}=\inf \left(x: 1-F(x) \leqslant \frac{1}{n}\right), \quad d_{n}=R\left(c_{n}\right) .
$$

Соотношение (9) приводится к виду

$$
\mathbf{P}\{\nu>n-k+1\}=\mathbf{P}\left\{L_{(n-k+1)}^{*} d_{n} \leqslant S_{m}^{*} B_{m}+A_{m}-c_{n}\right\},
$$

где $c_{n}$ и $d_{n}$ выбраны в соответствии с (19).

Из (20), центральной предельной теоремы и соотношений (16)-(18) получим, что при условии $1^{\circ}$

$$
\begin{aligned}
\mathbf{P}\{\nu>n-k+1\} & \longrightarrow \mathbf{P}\left\{\xi_{k} \leqslant b \eta+c\right\}=\int_{-\infty}^{+\infty} \mathbf{P}\left\{\xi_{k} \leqslant b x+c\right\} d \Phi(x) \\
& =\sum_{i=0}^{k-1} \int_{-\infty}^{+\infty} \Pi_{i}\left(e^{-b x-c}\right) d \Phi(x) .
\end{aligned}
$$

Если же выполнено условие $2^{\circ}$, то соответствуюшее утверждение легко следует из (20).

Д о к а 3 а т е л ь с т в о с л е I с т в и я 3.1. Нетрудно проверить, что в условиях следствия $\beta(F)=+\infty, R(t) \equiv 1, c_{n}=\log n, d_{n}=1$, а условие $1^{\circ}$ сводится к ограничениям: $\log n=o(\rho), m / \rho-\log n \longrightarrow c$.

4.2. Интегральные теоремы. Регулярный случай. В теоремах 2 и 3 рассмотрены случаи, когда размер эпидемии либо конечен с вероятностью 1 , либо эпидемия охватывает практически всю популяцию за исключением конечного числа восприимчивых индивидуумов. 
Перейдем теперь к рассмотрению центральной области, или регулярного случая, когда $k \sim n \alpha, 0<\alpha<1$.

Теорема 4. Пусть өыполнено (L), а также следующие два условuя:

$1^{\circ} . \quad A_{m} \rightarrow x_{0}$, причем $\left(A_{m}-x_{0}\right) \sqrt{n} \longrightarrow a_{0}$ и существует $F^{\prime}\left(x_{0}\right)>0$;

$2^{\circ} . \quad B_{m} d_{n}^{-1} \rightarrow b<\infty$, где $d_{n}^{2}=\alpha(1-\alpha)\left[\sqrt{n} F^{\prime}\left(x_{0}\right)\right]^{-2}, \alpha=F\left(x_{0}\right)$.

Тогда для любого фиксированного $x$ при $n \rightarrow \infty$

$$
\mathbf{P}\left\{\frac{\nu-n \alpha}{\sqrt{n \alpha(1-\alpha)}}>-x\right\} \stackrel{d}{\longrightarrow} \int_{-\infty}^{+\infty} \Phi(b u+c+x) d \Phi(u),
$$

где $c=a_{0} F^{\prime}\left(x_{0}\right)[\alpha(1-\alpha)]^{-1 / 2}$.

Если же $B_{m} d_{n}^{-1} \longrightarrow \infty,\left(A_{m}-x_{0}\right) B_{m}^{-1} \longrightarrow a_{0}, \quad B_{m}=O\left(n^{1 / 2}\right)$, mo $\mathbf{P}\left\{\nu>n \alpha-B_{m} x\right\} \stackrel{d}{\longrightarrow} \Phi\left(a_{0}+x\right)$.

Следствие 4.1. $E с л и T_{i} \sim \exp (\rho), L_{i} \sim \exp (1)$ и выполнены условия $m / \rho \rightarrow x_{0},\left(m / \rho-x_{0}\right) \sqrt{n} \longrightarrow a_{0}, n / m \rightarrow y_{0}, m o$

$$
\mathbf{P}\{\nu>n \alpha-\sqrt{n \alpha(1-\alpha)} x\} \stackrel{d}{\longrightarrow} \int_{-\infty}^{+\infty} \Phi(b u+c+x) d \Phi(u),
$$

где

$$
\alpha=1-e^{-x_{0}}, \quad b=F^{\prime}\left(x_{0}\right)\left[\frac{y_{0} x_{0}^{2}}{\alpha(1-\alpha)}\right]^{1 / 2}, \quad c=\frac{a_{0} F^{\prime}\left(x_{0}\right)}{\sqrt{\alpha(1-\alpha)}} .
$$

Ecsu же $n / m \longrightarrow \infty u\left(m / \rho-x_{0}\right) \sqrt{m} \longrightarrow a_{0}, \operatorname{mo} \mathbf{P}\{\nu>n \alpha\} \longrightarrow \Phi\left(a_{0}\right)$.

Док а з а т е ль с т в о т е о ре м ы 4 . Пусть $k=n \alpha-b_{n} x$, где $0<\alpha<1$ и $b_{n}=O(\sqrt{n})$. Если функция $F(x)$ дифференцируема в точке $x_{0}$ и $F^{\prime}\left(x_{0}\right)>0$, то в силу теоремы 5.8 из [20]

$$
L_{(k)}^{*} \equiv\left(L_{(k)}-c_{n}\right) d_{n}^{-1} \stackrel{d}{\longrightarrow} \xi
$$

где

$$
F_{\xi}(x)=\Phi(x), \quad c_{n}=x_{0}+\frac{k-n \alpha}{n F^{\prime}\left(x_{0}\right)}, \quad \alpha=F\left(x_{0}\right), \quad d_{n}^{2}=\frac{\alpha(1-\alpha)}{\left[n F^{\prime}\left(x_{0}\right)\right]^{2}} .
$$

Тогда согласно (9)

$$
\begin{aligned}
\mathbf{P} & \left\{\nu>n \alpha-b_{n} x\right\}=\mathbf{P}\left\{L_{(k+1)}^{*} \leqslant S_{m}^{*} B_{m} d_{n}^{-1}+\left(A_{m}-c_{n}\right) d_{n}^{-1}\right\} \\
& =\mathbf{P}\left\{L_{(k+1)}^{*} \leqslant S_{m}^{*} B_{m} d_{n}^{-1}+\left(A_{m}-x_{0}\right) \frac{\sqrt{n} F^{\prime}\left(x_{0}\right)}{\sqrt{\alpha(1-\alpha)}}-\frac{k-n \alpha}{\sqrt{n \alpha(1-\alpha)}}\right\} .
\end{aligned}
$$

Отсюда, из условий теоремы и соотношения (21) следует, что

$$
\mathbf{P}\left\{\nu>n \alpha-b_{n} x\right\} \stackrel{d}{\longrightarrow} \mathbf{P}\{\xi \leqslant b \eta+c+x\}=\int_{-\infty}^{+\infty} \Phi(b u+c+x) d \Phi(u) .
$$


3 ам еч ан и е. Если $B_{m} d_{n}^{-1} \longrightarrow \infty,\left(A_{m}-x_{0}\right) B_{m}^{-1} \longrightarrow a_{0}, B_{m}=$ $O(\sqrt{n})$, то

$$
\mathbf{P}\left\{\nu>n \alpha-B_{m} x\right\} \stackrel{d}{\longrightarrow} \Phi\left(a_{0}+x\right) .
$$

Доказательство следствия 4.1. Достаточно найти лишь значение параметра $b$ :

$$
\begin{aligned}
b & =\lim _{n \rightarrow \infty} B_{m} d_{n}^{-1}=\lim _{n \rightarrow \infty} \frac{\sqrt{m} / \rho}{\sqrt{\alpha(1-\alpha)} / \sqrt{n} F^{\prime}\left(x_{0}\right)} \\
& =\lim _{n \rightarrow \infty} F^{\prime}\left(x_{0}\right)\left[\left(\frac{m}{\rho}\right)^{2} \frac{n}{m} / \alpha(1-\alpha)\right]^{1 / 2}=x_{0} F^{\prime}\left(x_{0}\right) \sqrt{\frac{y_{0}}{\alpha(1-\alpha)}}
\end{aligned}
$$

4.3. Промежуточные случаи. Теперь опишем асимптотическое поведение в промежуточных случаях, когда $k \rightarrow \infty$, но $k / n \rightarrow 0$, или ког да $n-k \rightarrow \infty$, но $(n-k) / n \rightarrow 0$.

Теорема 5. Пусть $F(0)=0, F(x)$ непрерывна в $\left[0, \delta_{n}\right]$ u дифференцируема в $\left(0, \delta_{n}\right)$ для некоторого $\delta_{n}>0$, причем $F^{\prime}(+0)>0$. Если $A_{m} \rightarrow 0, n F\left(A_{m}\right) \rightarrow \infty, n B_{m}^{2} / F\left(A_{m}\right) \longrightarrow b_{0}^{2} \leqslant \infty$ и выполнено условие (L), mo

$$
\mathbf{P}\left\{\nu>n F\left(A_{m}\right)-b_{n} x\right\} \stackrel{d}{\longrightarrow} \begin{cases}\int_{-\infty}^{+\infty} \Phi\left(b_{0} u+x\right) d \Phi(u), & 0<b_{0}<\infty \\ \Phi(x), & b_{0}=0 \cdot \text { uлu } \infty\end{cases}
$$

ade

$$
b_{n}= \begin{cases}\left(n F\left(A_{m}\right)\right)^{1 / 2}, & b_{0}<\infty \\ n B_{m} F^{\prime}(+0), & b_{0}=\infty\end{cases}
$$

Следствие 5.1. Пусть $T_{i} \sim \exp (\rho), L_{i} \sim \exp (1)$. Если выполнень условия: $m=o(\rho), m n / \rho \rightarrow \infty, n / \rho \rightarrow b_{0} \leqslant \infty$, mо

$$
\mathbf{P}\left\{\nu>\frac{m n}{\rho}-\sqrt{\frac{m n}{\rho}} x\right\} \stackrel{d}{\longrightarrow} \begin{cases}\int_{-\infty}^{+\infty} \Phi\left(b_{0} u+x\right) d \Phi(u), & 0<b_{0}<\infty \\ \Phi(x), & b_{0}=0 \text { или } \infty\end{cases}
$$

Доказательст в о те о ремы 5. Как и ранее, используя (9), запишем

$$
\mathbf{P}\{\nu>k\}=\mathbf{P}\left\{L_{(k+1)}^{*} \leqslant S_{m}^{*} B_{m} d_{n}^{-1}+\left(A_{m}-c_{n}\right) d_{n}^{-1}\right\}
$$

где $c_{n}=F^{-1}(k / n), d_{n}=F^{-1}(k / n+\sqrt{k} / n)-F^{-1}(k / n)$.

При таком выборе нормирующих последовательностей $c_{n}$ и $d_{n}$ и в предположении, что $k \rightarrow \infty, k=o(n)$, из результатов Н. В. Смирнова $[18$, теорема 1] следует, что

$$
\mathbf{P}\left\{L_{(k+1)}^{*}<x\right\} \stackrel{d}{\longrightarrow} \Phi(x)
$$


при дополнительном условии

$$
\lim _{n \rightarrow \infty} \frac{F\left(c_{n}+d_{n} x\right)-F\left(c_{n}\right)}{\sqrt{k} / n}=x \quad \text { для любого } \quad x \in \mathbf{R} .
$$

Покажем, что при наших предположениях относительно $F(x)$ это условие выполнено.

В силу теоремы Лагранжа о конечных прирашениях имеем: при $x>0$

$$
\begin{aligned}
& \frac{F\left(c_{n}+d_{n} x\right)-F\left(c_{n}\right)}{\sqrt{k} n^{-1}}=\frac{F\left(c_{n}+d_{n} x\right)-F\left(c_{n}\right)}{d_{n} x} \frac{d_{n} x}{\sqrt{k}} n \\
& =x F^{\prime}\left(\theta_{n 1}\right) \frac{F^{-1}(k / n+\sqrt{k} / n)-F^{-1}(k / n)}{\sqrt{k} / n}=x \frac{F^{\prime}\left(\theta_{n 1}\right)}{F^{\prime}\left(F^{-1}\left(\theta_{n 2}\right)\right)},
\end{aligned}
$$

где $c_{n}<\theta_{n 1}<c_{n}+d_{n} x, k / n<\theta_{n 2}<k / n+\sqrt{k} / n$.

Переходя к пределу в этом равенстве, получим условие (24). Если же $x<0$, то $c_{n}+d_{n} x<\theta_{n 1}<c_{n}$ и все сохраняет силу, если учесть, что $d_{n}=o\left(c_{n}\right)$.

Пусть теперь $k=n F\left(A_{m}\right)-b_{n} x$, где $b_{n}=o\left(n F\left(A_{m}\right)\right)$. Тогда утверждение (23), очевидно, имеет место, и остается найти асимптотическое поведение $\Delta_{n}(x)=\left(A_{m}-c_{n}\right) d_{n}^{-1}$. Снова пользуясь теоремой Лагранжа, получим

$$
\begin{aligned}
\Delta_{n}(x) & =\frac{F^{-1}\left(F\left(A_{m}\right)-b_{n} x / n\right)-F^{-1}\left(F\left(A_{m}\right)\right)}{F^{-1}(k / n+\sqrt{k} / n)-F^{-1}(k / n)} \\
& =\frac{b_{n} x}{n F^{\prime}\left(F^{-1}\left(\theta_{n 3}\right)\right)} \frac{F^{\prime}\left(F^{-1}\left(\theta_{n 4}\right)\right)}{\sqrt{k}} n \\
& =\frac{F^{\prime}\left(F^{-1}\left(\theta_{n 4}\right)\right)}{F^{\prime}\left(F^{-1}\left(\theta_{n 3}\right)\right)} \frac{b_{n} x}{\sqrt{n F\left(A_{m}\right)-b_{n} x}}
\end{aligned}
$$

Выбирая $b_{n}=\sqrt{n F\left(A_{m}\right)}$ и переходя к пределу, с учетом того, что $\theta_{n 3}$ и $\theta_{n 4}$ стремятся к нулю, получим, что

$$
\Delta_{n}(x) \sim x .
$$

Утверждение теоремы при $b_{0}<\infty$ теперь слецует из центральной предельной теоремы и соотношений $(22),(23)$ и $(25)$. Случай $b_{0}=\infty$ легко следует из (22).

\section{СПИСОК ЛИТЕРАТУРЫ}

1. Баруча-Рид A. T. Элементы теории марковских процессов и их приложения. М.: Наука, 1969, 511 c.

2. Weis G. On the spread of epidemics by carries. - Biometrics, 1965, v. 21, № 2, p. $481-490$. 
3. Севастьянов Б. А. Ветвящиеся процессы с взаимодействием частиц. - В сб.: Тезисы докладов III Международной Вильнюсской конференции по теории вероятностей и математической статистике. Т. II. Вильнюс: Ин-т математики и кибернетики АН ЛитССР, 1981, с. 139-140.

4. Gani J. On the partial differential equation of epidemic theory. I. - Biometrika, 1965 , v. 52, № 3-4, p. 617-622.

5. Siskind V. Solution of the general stochastic epidemic. - Biometrika, 1965, v. 52, № 3-4, p. 613-616.

6. Woods A. J. First-passage times for the partial sums of a sequence of geometric distribution. - J. Appl. Probab., 1982, v. 19, p. 430-432.

7. Стариев $A$. H. О распределении момента поглощения для одного класса двумерных марковских случайных блужданий. - Узб. матем. журн., 1994, № 4, c. $80-88$.

8. Bailey N. T. J. Some problems in the statistical analysis of epidemic data. - J. Roy. Statist. Soc., Ser. B, 1955, v. 17, p. 35-38.

9. Whittle $P$. The outcome of stochastic epidemic. - Biometrika, 1955, v. 42, № 42, p. 116-122.

10. Нагаев A. B., Стариев A. Н. Асимптотический анализ одной стохастической модели эпидемий. - Теория вероятн. и ее примен., 1970, т. XV, в. 1, с. 97-105.

11. Стариев $A$. H., Чай 3. C. Предельные теоремы для размера эпидемии в одной обобщенной вероятностной модели. - В сб.: Вероятностные модели и математическая статистика. Ташкент: Фан, 1987, с. 92-105.

12. Старчев $A$. Н. Предельные теоремы для размера эпидемии в общей вероятностной модели. - В сб.: Случайные процессы и статистические выводы. Ташкент: Фан, 1971, с. 60-73.

13. Sellke $T$. On the asymptotic distribution of the size of a stochastic epidemic. J. Appl. Probab., 1983, v. 20, p. 390-394.

14. Ball $F$. A unified approach to the distribution of total size and total area under the trajectory of infectives in epidemic models. - Adv. Appl. Probab., 1986, v. 18, p. $281-310$.

15. Кендалл M., Стюарт A. Теория распределений. М.: Наука, 1966, 587 с.

16. Gnedenko $B$. V. Sur la distribution limite du terme maximum d'une série aléatoire. Ann. Math., 1943, v. 44, p. 423-4.53.

17. Смирнов $H$. В. Предельные законы распределения для членов вариационного ряда. - Труды матем. ин-та им. В. А. Стеклова АН СССР, 1949, т. 25, с. 1-60.

18. Смиряов $H . B$. Некоторые замечания о предельных законах для членов вариационного ряда. - Теория вероятн. и ее примен., 1967, т. XII, в. 2, с. 391-392.

19. Галамбош Я. Асимптотическая теория экстремальных порядковых статистик. М.: Наука, 1984, $304 \mathrm{c}$.

20. Balkema A. A., de Haan L. Limit distributions for order statistics. II. - Teopux вероятн. и ее примен., 1978, т. XXIII, в. 2, с. 358-375. 